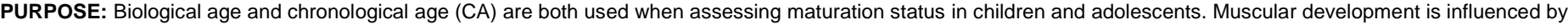
puberty and somatic maturity, an indicator of biological age, which can be estimated by the age at peak height velocity (PHV). However, it is unclear whether muscle morphology is influenced more by biological or chronological age in young judo athletes.

METHODS: Anthropometric data [weight (BW) and standing and seated height] were collected from thirty-three youth judo athletes [males: $n=23$, females: $n=10 ;$ CA:

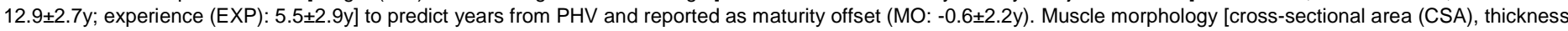
(MT), pennation angle (PA), fascicle length (FL), and echo-intensity (EI)] of the dominant and non-dominant vastus lateralis (VL) was assessed via ultrasonography. The average of both legs was calculated for each morphological measure. Data were examined using stepwise regression to determine if CA, MO, or EXP were predictive of muscle morphology. Partial correlations were used to determine relationships among the identified predictive measures and muscle morphology.

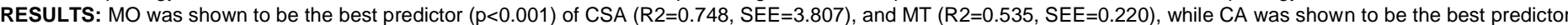

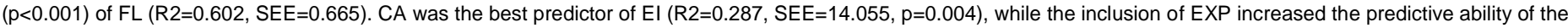

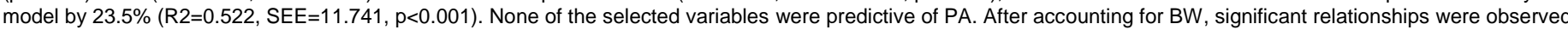

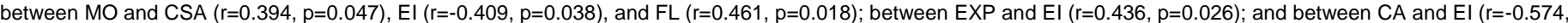
$\mathrm{p}=0.002)$ and $\mathrm{FL}(\mathrm{r}=0.504, \mathrm{p}=0.009)$

CONCLUSION: Biological age (i.e., maturity offset) appears to be indicative of lower body muscle size, whereas chronological age, and potentially years of experience,

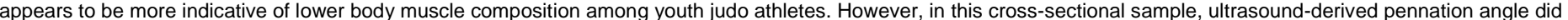
not appear to be related to age, maturation status, or years of experience.

Board \#2 June 1, 2:00 PM - 3:30 PM

\title{
Muscle Strength Is Significantly Associated With Calcaneal Bone Mineral Density Among Children And Adolescents From Colombia: A Cross-sectional Study
}

Monica Liliana Ojeda-Pardo ${ }^{1}$, Luis Andrés Téllez Tinjaca ${ }^{1}$, Javier Martínez-Torres ${ }^{1}$, Diogo Rodrigues-Bezerra ${ }^{2}$, Jorge Enrique CorreaBautista ${ }^{2}$, Robinson Ramírez-Vélez ${ }^{2} .{ }^{1}$ Universidad Santo Tomas, Bogotá D.C, Colombia. ${ }^{2}$ Universidad del Rosario, Bogotá D.C, Colombia. Email: robin640@hotmail.com

(No relationships reported)

PURPOSE: Dual energy X-ray absorptiometry (DEXA) is currently the gold standard for the assessment of bone mineral density (BMD). Quantitative ultrasound (QUS), on the other hand, is a radiation-free alternative for the assessment of bone strength in the paediatric population. Relationship between BMD and muscular fitness outcomes in children and adolescents have not been published in a Latin-American population. In this cross-sectional study we aimed to investigate the association of muscular fitness with $\mathrm{BMD}$ at the calcaneal in Colombia children and adolescent aged 9 to 17.9 years.

METHODS: Cross sectional study. A sample of 1,001 healthy Colombian youth (boys $n=445$ and girls $n=556$ ), participated in the study. BUA (in decibels per megahertz) was measured using Achilles device (Lunar, Madison, WI). Three components of field-based muscular fitness were measured: handgrip, vertical jump and standing long jump test. RESULTS: After adjusting for sex and age, we found a significant association of handgrip with BMD at calcaneal $(r=0.651)$. Furthermore, we observed significant associations of vertical jump $(r=0.341)$ and standing long jump $(r=0.415), p<.001$ with BMD.

CONCLUSIONS: We found that muscular fitness was associated with BMD in children and adolescents. Further prospective studies are needed to further investigate the association between muscular fitness and BMD in youth population.

Funding COLCIENCIAS (Contract № 671-2014 Code 122265743978).

Board \#3 June 1, 2:00 PM - 3:30 PM

Proprioception Changes After Eccentric Exercise Of The Elbow Flexors: Comparison Between Children And Adults

Trevor C. Chen ${ }^{1}$, Ming-Ru Lin ${ }^{2}$, Chih-Chiao Ho ${ }^{1}$, Hsin-Lian Chen ${ }^{3}$, Kuo-Wei Tseng ${ }^{4}$, Kazunori Nosaka ${ }^{5} .{ }^{1}$ National Taiwan Normal University, Taipei City, Taiwan. ${ }^{2}$ National Chiayi University, Chaiyi County, Taiwan. ${ }^{3}$ National Chiayi University, Chiayi County, Taiwan. ${ }^{4}$ University of Taipei, Taipei City, Taiwan. ${ }^{5}$ Edith Cowan University, Western Australia, Australia.

Email: tcchen@ntnu.edu.tw

(No relationships reported)

Significant impairment of proprioception such as position sense (PS) and joint reaction angle (JRA) was observed after the initial bout of unilateral eccentric exercise (ECC) of the knee flexors (KF), but the second of the same exercise performed 3 weeks later induced smaller changes in PS and JRA (Paschalis et al. EJAP 2008). Chen et al. (EJAP 2014) reported that the magnitude of muscle damage after unilateral ECC of the elbow flexors (EF) was smaller for children than adult men. However, no previous study has compared between children and adults for PS and JRA changes after ECC.

PURPOSE: The present study compared changes in several indirect markers of muscle damage and proprioception parameters after maximal EF ECC between children and adult women, and between two ECC bouts (ECC1 and ECC2).

METHODS: Thirteen girls (9-10 y) and young untrained women (20-24 y), respectively, performed two bouts (separated by 2 weeks) of 5 sets of 6 EF eccentric contractions by lowering a dumbbell $\left(60 \%\right.$ of pre-exercise maximal voluntary isometric contraction strength: MVC) with the non-dominant arm from an elbow flexed $\left(90^{\circ}\right)$ to a fully extended position. Changes in maximal voluntary isokinetic concentric contraction strength (MVC-CON), muscle soreness (SOR), plasma creatine kinase (CK) activity, PS and JRA of the elbow were measured before, immediately after and 1-5 days after each exercise. Changes in these variables over time were compared between groups, and between bouts by a mixed model two-way ANOVA.

RESULTS: Significant changes in all variables were found after ECC1 for both groups, but the changes were significantly smaller for girls (peak changes, MVC-CON: $-15 \pm$ $3 \%$, PS: $1.5 \pm 0.3^{\circ}$ and JRA: $-1.7 \pm 0.4^{\circ}$ ) than adult women (MVC-CON: $-30 \pm 4 \%$, PS: $4.5 \pm 0.6^{\circ}$, JRA: $-4.1 \pm 0.7^{\circ}$ ). Changes in all variables were smaller after ECC2 than ECC1 $\left(P<0.05\right.$ ) for both girls (MVC: $-12 \pm 2 \%$, PS: $0.4 \pm 0.3^{\circ}$, JRA: $-1.2 \pm 0.2^{\circ}$ ) and adult women (MVC-CON: $-21 \pm 3 \%, P S: 2.6 \pm 0.3^{\circ}$ and JRA: $-2.5 \pm 0.4^{\circ}$ ), and the changes were still smaller for children than adult women $(P<0.05)$

CONCLUSIONS: These results show that the magnitude of proprioception impairment was associated with the magnitude of muscle damage, and confirmed the previous study (Chen et al. 2014) that children were less susceptible to muscle damage than adults. Supported by MOST, Taiwan (MOST103-2410-H-003-114).

\section{June 1, 2:00 PM - 3:30 PM \\ Board \#4 \\ Limiters to Rapid Repetitive Tapping in Young and Older Adults}

Erica Hartman, Catherine Dollard, Michael Busa, Jane Kent, FACSM. University of Massachusetts, Amherst, Amherst, MA. Email: elhartman@kin.umass.edu

(No relationships reported)

A brief Foot Tap Test (FTT, 10s of rapid tapping) relies on rapid, repetitive activation of the dorsiflexor muscles and has been shown to be slower in older adults. The source of this slowing is not known, but could include: greater performance variability, slowed central and/or peripheral activation, or slowed muscle contractile properties. 